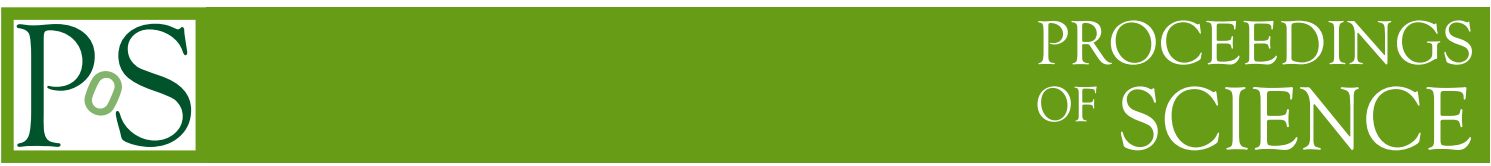

\title{
Universal Landau Pole at the Planck scale *
}

\author{
Alexander A. Andrianov ${ }^{\dagger a, b}$, \\ ${ }^{a}$ V.A.Fock Department of Theoretical Physics, \\ Saint Petersburg State University, Saint Petersburg, Russia \\ E-mail: andrianov@icc.ub.edu \\ Domenec Espriu $^{b}$, Maxim A. Kurkov ${ }^{c, d}$ and Fedele Lizzi ${ }^{b, c, d}$ \\ ${ }^{b}$ Departament ECM and Insitut de Ciencies del Cosmos(ICC), \\ University of Barcelona, Barcelona, Spain \\ ${ }^{c}$ Dipartimento di Fisica, Università di Napoli Federico II, Napoli, Italia \\ ${ }^{d}$ INFN Sezione di Napoli, Napoli, Italia \\ E-mail: espriu@icc.ub.edu, fedele.lizzi@na.infn.it,kurkov@na.infn.it
}

\begin{abstract}
The concept of quantum gravity entails that the usual geometry loses its meaning at very small distances and therefore the grand unification of all gauge interactions with the property of asymptotic freedom happens to be questionable. We propose an unification of all gauge interactions in the form of an "Universal Landau Pole" (ULP), at which all gauge couplings diverge (or, better to say, become very strong). We show that the Higgs quartic coupling also substantially increases whereas the Yukawa couplings tend to zero. Such a singular (or strong coupling) unification is obtained after adding to the Standard Model matter more fermions with vector gauge couplings and hypercharges identical to the SM fermions. The influence of new particles also may prevent the Higgs quartic coupling from crossing zero, thus avoiding the instability (or metastability) of the SM vacuum. As well this fermion pattern opens a way to partially solve the hierarchy problem between masses of quarks and leptons.
\end{abstract}

The XXI International Workshop High Energy Physics and Quantum Field Theory June 23 - June 30, 2013

Saint Petersburg Area, Russia

*A.A., D.E. and F.L. are partially supported by projects FPA2010-20807, 2009SGR502 and CPAN (Consolider CSD2007-00042). A.A. is also supported by Grant RFBR 13-02-00127 and by SPbSU grant 11.38.660.2013.

†Speaker. 


\section{Outline}

At high energies and momentum transfers the coupling constants of fundamental gauge interactions behave differently [1]. While the couplings of weak and strong interactions decrease (with different speed) as the energy increases, the coupling of the abelian hypercharge interaction related to electromagnetic forces grows unboundedly, and eventually diverges, a behavior referred as Landau pole [2].

The incorporation of new particles modifies this behavior. From the empirical crossing of gauge couplings at high energies an appealing idea was set forth that the three interactions coincided at a single Grand Unification scale, and this was interpreted to signal the presence of a non-abelian GUT group.

On the other hand, continuing the flow of the three couplings beyond the previous scales would give rise to the running of the hypercharge coupling and its divergence at the finite scale of $10^{53} \mathrm{GeV}$ : for a non asymptotic free theory at some energy there will be a pole.

The behavior of the quartic Higgs coupling $\lambda$ and of the Yukawa couplings, especially of the largest $t$ quark one, under the renormalization flow is also important at high energy. They both decrease, but while $y_{t}$ remains positive, $\lambda$ becomes negative in the presence of a relatively light Higgs with mass $\sim 126 \mathrm{GeV}$ [3]. This signals an instability (metastability) of the theory $[4,5]$.

The unification of the forces with enlargement of the gauge symmetry to a grand unified nonabelian group guarantees the presence of asymptotic freedom and provides the ability to describe particles and fields at arbitrarily small distances. However, in nature there is also gravity and when energies and momenta approach the Planck scale the quantum gravity effects will certainly alter the picture. Typically models of quantum gravity (see e.g. $[6,7,8]$ ) prescribe a minimal point separation below which the notion of length may not exist. Then it is not obvious why one should expect quantum field theory to remain perturbatively valid at or beyond the Planck scale. Then the necessity for asymptotic freedom at the fundamental scale of space-time geometry is a disputable matter.

In our talk we outline an alternative way to treat gauge interactions at a level of the Planck scale (based on[9]) and elucidate the following topics:

- Do we really need asymptotic freedom?

Our understanding of quantum gravity suggests that at the Planck scale the usual geometry loses its meaning. Then grand unification in a large non-abelian group naturally endowed with the property of asymptotic freedom may also lose its motivation.

- Singular unification:

an unification of all fundamental interactions at the Planck scale in the form of a Universal Landau Pole (ULP), at which all gauge couplings diverge.

- Minimal working model of the Universal Landau Pole.

The unification is achieved with the addition of fermions with vector gauge couplings coming in multiplets and with hypercharges identical to those of the Standard Model. 
- Stability of the Higgs Potential.

The Higgs quartic coupling remains positive and diverges while the Yukawa couplings vanish.

- Softening a hypothetical Landau pole

down to very strong couplings in the presence of an ultraviolet fixed point of gauge coupling RG induced by gravity.

\section{Do we really need asymptotic freedom?}

Let us formulate pro and contra of the GUT unification:

- Simplicity: the less parameters we have the better predictions we do $\rightarrow$ unification.

- Asymptotic freedom (flat space-time): the theory is valid up to infinitely high energies.

- BUT what about gravity?

At the energies of order of Planck scale $M_{\mathbf{P I}} \sim 10^{19} \mathrm{GeV}$ gravity becomes strongly coupled, concept of weakly interacting point-like fields looses its meaning!

- Simplicity + pointless geometry $\rightarrow$ singular unification.

\subsection{Singular unification: Universal Landau Pole}

We propose a singular unification at the Planck scale: one should find such a generalization of the Standard Model, that under the renormalization group flow ALL gauge couplings meet their common Landau pole at the Planck scale.

$$
g_{1,2,3}(\mu) \rightarrow \infty \text { at } \mu \rightarrow M_{P l}
$$

Correspondingly kinetic terms of ALL gauge fields vanish and they cannot propagate anymore.

$$
-\frac{1}{4 g_{i}(\mu)^{2}} F_{\mu v}^{i} F_{i}^{\mu v} \rightarrow 0 \text { at } \mu \rightarrow M_{P l}
$$

We are looking for a minimal generalization of the SM that satisfy the properties of:

- Simplicity:

the gauge group of SM $S U(3) \times S U(2) \times U(1)$. We add only fermions. Enlarging the gauge group in principle could be motivated by introduction of a GUT group. However it leads to ULP at $10^{\mathbf{1 6}} \mathrm{GeV}$ (see [10] for a review) much smaller than $\mathbf{M}_{\mathbf{P I}}$.

- Higgs sector to remain unchanged.

If new fermions are described by 4-component spinors with $S U(2)$ singlet Dirac masses and vector-like gauge interactions there is no necessity for any Higgs fields. It fits well the recent LHC bounds on the number of generations [11].

- NO pathological electric charge

which results in restrictions on the representations of new fermions. 
- Stability:

the quartic coupling of the Higgs field self interaction $\lambda$ is always positive under the renormalization group flow. It discriminates a single scenario with four generations.

\subsection{Minimal working ULP: realization}

We use Dirac mass terms $M \bar{\psi} \psi$ for new fermions and we are looking for a minimal number of them. New fermions belong to known representations of gauge group.

L-quarkons: $\mathrm{SU}(3)$ - triplets, $\mathrm{SU}(2)$ - doublets, $Y=\frac{1}{3}$

R-quarkons: $\mathrm{SU}(3)$ - triplets, $\mathrm{SU}(2)$ - singlets, $Y=\frac{4}{3},-\frac{2}{3}$

L-leptos: $\mathrm{SU}(3)$ - singlets, $\mathrm{SU}(2)$ - doublets, $Y=-1$

R-leptos: $\mathrm{SU}(3)$ - singlets, $\mathrm{SU}(2)$ - singlets, $Y=-2,0$

We notice that the L- and R- notations do not imply left and right chiralities! They are vectorlike relatives. Thus the only new vertexes appearing in the theory couple Quarkons and Leptos to E-W gauge bosons and gluons:
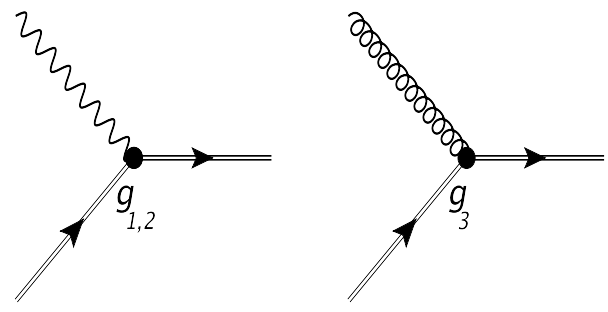

Therefore at one loop level only the beta functions of gauge fields are modified. The ULP can be rendered within four identical "generations" of new vector-like massive fermions with different mass scales:

- At 5.0 $\cdot \mathbf{1 0}^{\mathbf{3}} \mathrm{GeV}$ L-quarkons $\left(N_{\mathbf{L}-\text { quarkon }}=4\right)$.

- At $3.7 \cdot 10^{7} \mathrm{GeV}$ R-quarkons $\left(N_{\mathbf{R}-\text { quarkon }}=4\right)$.

- At $2.6 \cdot 10^{14} \mathrm{GeV} \mathrm{L}$ and R-leptos $\left(N_{\mathbf{L}-\text { leptos }}=N_{\mathbf{R}-\text { leptos }}=4\right)$.

This result follows from optimization of the RG running to the low-energy SM group and to the ULP at about $10^{19} \mathrm{GeV}$.

At one loop the running of the couplings is given by simple equations

$$
\frac{d g_{i}(t)}{d t}=\beta_{i}(t), \quad \beta_{i} \equiv \frac{1}{16 \pi^{2}} g_{i}^{3} b_{i}, \quad t \equiv \log \frac{\mu}{G e V} .
$$

Where $i=1,2,3$ represent the $\mathrm{U}(1), \mathrm{SU}(2)$ and $\mathrm{SU}(3)$ couplings respectively. The presence of new particles will alter this running. At one loop the behavior of inverse coupling constants $1 / \alpha_{i} \equiv 4 \pi / g_{i}^{2}$ are linear. The presence of new particles just alters the slope of straight lines. 
For the RG equations of gauge couplings, the constants $b_{i}$ are given by:

$$
\begin{gathered}
b_{1}=\frac{41}{6}+\frac{2}{3} N_{\mathbf{L}-\text { leptos }}+\frac{4}{3} N_{\mathbf{R}-\text { leptos }}+\frac{2}{9} N_{\mathbf{L}-\text { quarkon }}+\frac{4}{3} N_{\mathbf{R}-\text { quarkon }} . \\
b_{2}=-\frac{10}{3}+\frac{2}{3} N_{\mathbf{L}-\text { leptos }}+2 N_{\mathbf{L}-\text { quarkon }} . \\
b_{3}=-7+\frac{4}{3}\left(N_{\mathbf{L}-\text { quarkon }}+N_{\mathbf{R}-\text { quarkon }}\right)
\end{gathered}
$$

The integers $N$ in these formulas refer to the number of quarkon and leptos multiplets contributing to beta functions.

Since the coefficients are piecewise constant, and change at the energies representing the scale at which the new particles, it is possible to do a systematic search. We have imposed as boundary condition of the differential equation that $1 / \alpha_{i}=0$ at the Planck scale $m_{p}$. The other low energy boundary conditions are given by the experimental values: $\alpha_{s}=0.118, g_{1}=0.359, g_{2}=0.648$, $g_{3}=1.165, y=0.938, \lambda=0.126$ for $M_{H}=126 \mathrm{GeV}$ at the scale of the top mass $\mu=M_{t}=173 \mathrm{GeV}$.

We require that the scales be between the TeV region and the ULP, and that the evolution is monotonous (the curves must not intersect themselves). It results in the only allowed order of the different thresholds as one goes up: L-quarkons, R-quarkons, L-Leptos, R-Leptos and one finds the following solutions.
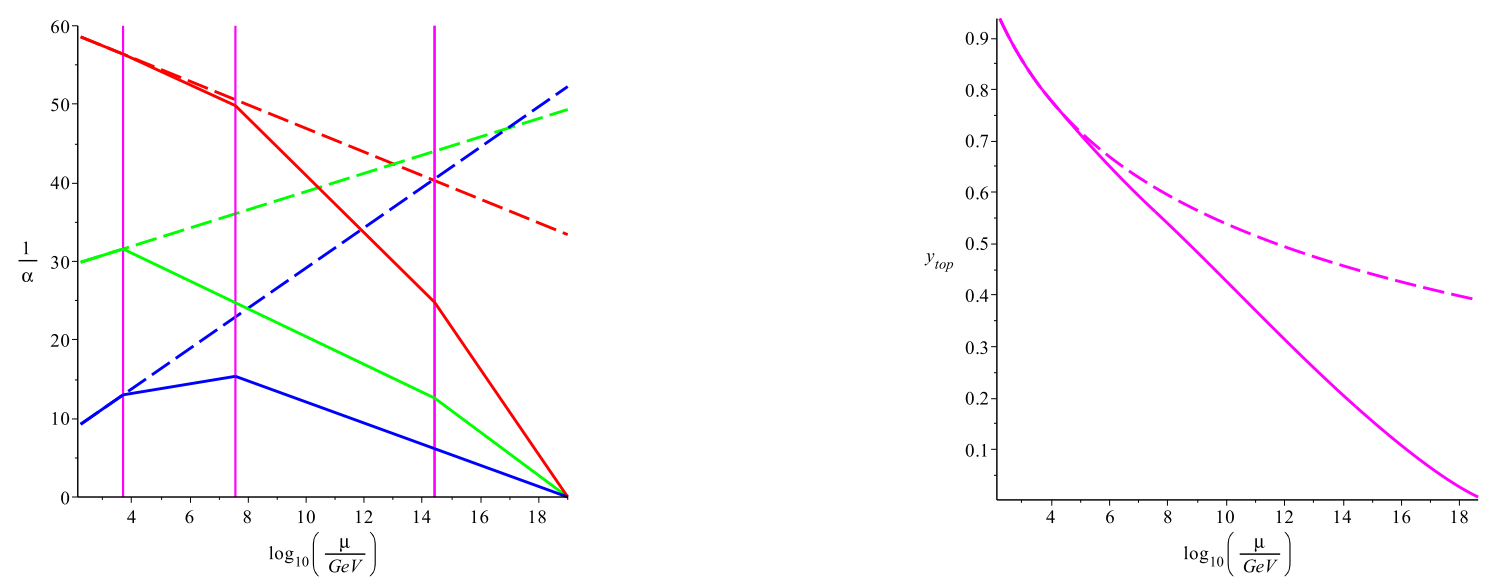

Figure 1: The running of gauge, $g_{1,2,3}$ (left plots) and of Yukawa, $y_{t}$ (right plot) coupling constants. The dotted lines are related to the SM case.

As far as the top Yukawa coupling is concerned the equation is

$$
\frac{d y(t)}{d t}=\frac{1}{(4 \pi)^{2}} y\left(-\frac{9}{4} g_{2}^{2}-\frac{17}{12} g_{1}^{2}-8 g_{3}^{2}+\frac{9}{2} y^{2}\right)
$$

This coupling happens to be almost undistinguishable from the standard model for energies up to $10^{6} \mathrm{GeV}$, and vanishes at the ULP. 


\section{On the stability of the Higgs potential}

Now we clarify how our vector-like fermions save the Universe from instability, i.e. how they prevent the RG flow from driving the quartic coupling $\lambda(\mu)$ to negative values.

$$
\frac{d \lambda(t)}{d t}=\frac{1}{16 \pi^{2}}\left(24 \lambda^{2}-6 y^{4}+\frac{3}{4} g_{2}^{4}+\frac{3}{8}\left(g_{2}^{2}+g_{1}^{2}\right)^{2}+\left(-9 g_{2}{ }^{2}-3 g_{1}{ }^{2}+12 y^{2}\right) \lambda\right) .
$$

The Yukawa constant term marked red is decreasing and the rest is positive keeping the coupling $\lambda(\mu)$ positive and growing.

The following figure demonstrates the one(two)-loop RG running of Higgs boson quartic coupling with a Landau pole driven by gauge couplings.

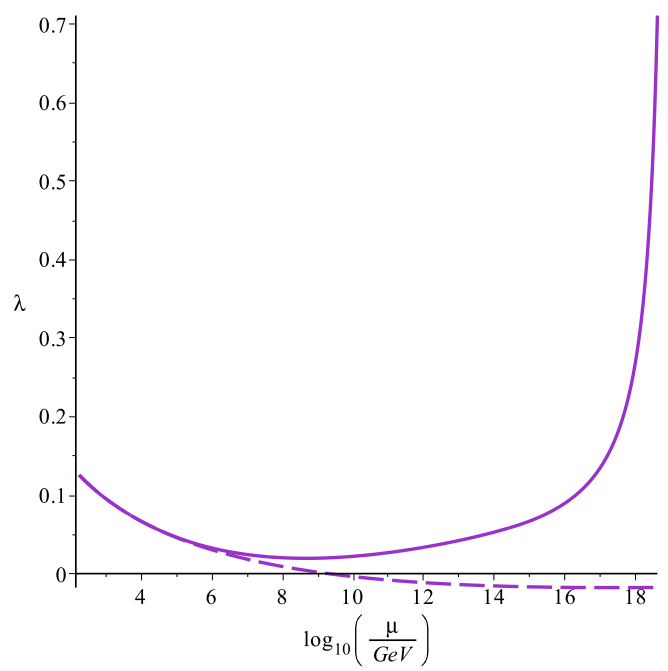

\section{UV completion}

It could well be the case that the onset of gravity corrections renders the ULP non-singular. Indeed gravity being non-renormalizable will require higher-dimensional operators with more derivatives to make the theory finite. In particular, we expect dimension six kinetic terms like

$$
\frac{\gamma}{2 M_{P}^{2}} \operatorname{tr}\left(D_{\mu} W^{\mu v} D_{\mu} W_{v}^{\mu}\right)+\cdots
$$

A more precise treatment can reckon upon an UV fixed point which leads to dimensional reduction of gauge fields,

$$
F_{\mu v}\left(\frac{1}{g(\mu)^{2}}+\gamma \frac{\square}{M_{P l}^{2}}+\cdots\right) F^{\mu v} \rightarrow \gamma F_{\mu v} \frac{\square}{M_{P l}^{2}} F^{\mu v} \text { at } \mu \rightarrow M_{P l}
$$

This would correspond to a renormalization of the gauge coupling induced by gravity of the form

$$
\frac{1}{g^{2}\left(p^{2}\right)} \simeq \beta_{0} \log \frac{m_{P}^{2}}{p^{2}}+\gamma \frac{p^{2}}{m_{P}^{2}}
$$

Thus gravitational corrections may soften the ULP behavior towards a new fixed point at strong coupling regime (see, for instance, [12] ). 


\section{Summary}

- An idea of singular unification of ALL gauge interactions at the Planck scale, can be realized in the form of the Universal Landau Pole (ULP).

- The minimal working model of ULP generalization of the SM is constructed.

- Under the RG flow the top Yukawa coupling eventually goes to zero while the quartic coupling has a concordant singularity at the Planck scale. Such a RG behavior saves the Universe from instability problem.

- Yukawa couplings for quarks and leptons run to zero differently: the latter for leptons are diminishing more slowly (due to lack of gluon contribution). Thereby the ULP unification may give a partial resolution of fermion mass hierarchy problem in the range of strong gauge couplings [13]. For this problem the two-loop contribution may be essential.

\section{References}

[1] J. Beringer et al. [Particle Data Group], Phys. Rev. D 86, 010001 (2012).

[2] L. D. Landau, A. A. Abrikosov, and I. M. Khalatnikov, Dokl. Akad. Nauk SSSR 95 (1954), 497, 773, 1177 ; L.D.Landau, I. Ya.Pomeranchuk, Dokl. Akad. Nauk SSSR 102 (1955) 489.

[3] G. Aad et al. [The ATLAS collaboration], Phys. Lett. B 716 (2012) 1; S.Chatrchyan et al. [The CMS collaboration], Phys. Lett. B716 (2012) 30.

[4] N. V. Krasnikov, Yad. Fiz. 28 (1978) 549; P.Q. Hung, Phys. Rev. Lett. 42 (1979) 873; H.D. Politzer and S. Wolfram, Phys. Lett. B 82 (1979) 242 [Erratum ibid. B83 (1979) 421].

[5] J. Elias-Miro, J. R. Espinosa, G. F. Giudice, G. Isidori, A. Riotto and A. Strumia, Phys. Lett. B 709 (2012) 222;

F. Bezrukov, M. Y. .Kalmykov, B. A. Kniehl and M. Shaposhnikov, JHEP 1210140 (2012).

[6] J. Alfaro, D. Espriu and D. Puigdomenech, Phys. Rev. D 86, 025015 (2012).

[7] A. Connes, M. Marcolli, Noncommutative Geometry, Quantum Fields and Motives, AMS 2007; P. Aschieri, M. Dimitrijevich, P. Kulish, F. Lizzi, J. Wess, Noncommutative Spacetimes, Springer Lecture Notes 774, 2009.

[8] C. Rovelli, Quantum Gravity, Cambridge University Press, 2007.

[9] A.A. Andrianov. D. Espriu, M.A. Kurkov and F. Lizzi, Phys. Rev. Lett. 111, 011601 (2013).

[10] V. A. Rubakov and S. V. Troitsky, Trends in grand unification: Unification at strong coupling and composite models, hep-ph/0001213.

[11] See e.g. A. Lenz, Advances in High Energy Physics 2013 (2013) 910275.

[12] M. Shaposhnikov and C. Wetterich, Phys. Lett. B 683 (2010) 196; M. E. Shaposhnikov, Theor. Math. Phys. 170 (2012) 229.

[13] A.A. Andrianov. D. Espriu, M.A. Kurkov and F. Lizzi, in preparation. 